\title{
The adverse effect of multiple comorbidity on functional outcome in patients with ischemic stroke or transient ischemic attack
}

Han-Yeong Jeong ${ }^{\mathrm{a}}$, Tae Jung Kim ${ }^{\mathrm{a}}$, Heejung $\mathrm{Mo}^{\mathrm{a}}$, Chan-Hyuk Lee ${ }^{\mathrm{a}}$, Yerim Kim

Sang-Bae Ko ${ }^{\mathrm{a}}$, Byung-Woo Yoon ${ }^{\mathrm{a}}$

${ }^{a}$ Department of Neurology, Seoul National University Hospital, Seoul, Republic of Korea

${ }^{b}$ Department of Neurology, Kangdong Sacred Heart Hospital, Hallym University College of Medicine, Seoul, Republic of Korea

\section{PURPOSE}

Multiple comorbidities have negative impact on functional outcomes in cardiovascular diseases including ischemic stroke. However, their individual association with acute ischemic stroke(AIS) or transient ischemic attack(TIA) remains to be elucidated. This study aims to evaluate the impact of comorbidity on functional outcome in patients with AIS or TIA.

\section{METHODS}

Study population: 2,449 AIS and 248 TIA patients within 7 days after symptom onset

Outcome variable: modified Rankin scale (mRS) at 3 months Excellent outcome (mRS 0 or 1)

vs. non-excellent outcome ( $\mathrm{mRS}>1)$

Statistical analysis: logistic regression analysis

To identify the comorbidity conditions

independently associated with functional outcomes

\begin{tabular}{|c|c|c|c|c|}
\hline \multicolumn{5}{|c|}{ RESULTS } \\
\hline \multicolumn{5}{|c|}{ Table 1. Basal characteristics of study population } \\
\hline \multicolumn{5}{|c|}{ Acute ischemic stroke } \\
\hline mean $\pm S D$ or $n(\%)$ & $\begin{array}{c}\text { All AIS } \\
\mathrm{n}=2,449\end{array}$ & $\begin{array}{c}\mathrm{mRS} \\
\mathrm{n}=1,371\end{array}$ & $\begin{array}{l}m R S \geq 2 \\
n=1,078\end{array}$ & $p$-value \\
\hline Age, years & $67.3 \pm 12.7$ & $64.8 \pm 12.7$ & $70.4 \pm 12.1$ & $<0.001$ \\
\hline Gender, male & $1,490(60.8)$ & $891(65.0)$ & $599(55.6)$ & $<0.001$ \\
\hline Body mass index, $\mathrm{kg} / \mathrm{m}^{2}$ & $23.5 \pm 3.3$ & $24.0 \pm 3.2$ & $22.9 \pm 3.3$ & $<0.001$ \\
\hline \multicolumn{5}{|l|}{ Stroke classification } \\
\hline Large artery disease & $791(32.3)$ & $441(32.2)$ & $350(32.5)$ & \\
\hline Small vessel occlusion & $478(19.5)$ & $337(24.6)$ & $141(13.1)$ & $<0.001$ \\
\hline Cardioembolism & $604(24.7)$ & $301(22.0)$ & $303(28.1)$ & \\
\hline Other etiology & $219(8.9)$ & $89(6.5)$ & $130(12.1)$ & \\
\hline Undetermined etiology & $357(14.6)$ & $203(14.8)$ & $154(14.3)$ & \\
\hline Initial NIHSS & $5.1 \pm 5.7$ & $2.9 \pm 3.5$ & $7.9 \pm 6.7$ & $<0.001$ \\
\hline Premorbid mRS & $0.8 \pm 1.4$ & $0.5 \pm 1.0$ & $1.2 \pm 1.7$ & $<0.001$ \\
\hline Discharge NIHSS & $3.9 \pm 6.9$ & $1.3 \pm 2.2$ & $7.3 \pm 9.0$ & $<0.001$ \\
\hline Discharge mRS & $1.8 \pm 1.6$ & $1.0 \pm 0.9$ & $2.9 \pm 1.5$ & $<0.001$ \\
\hline \multicolumn{5}{|c|}{ Transient ischemic attack } \\
\hline mean $\pm S D$ or $n(\%)$ & $\begin{array}{l}\text { All TIA } \\
n=248\end{array}$ & $\begin{array}{l}\mathrm{mRS}=0,1 \\
\mathrm{n}=216\end{array}$ & $\begin{array}{c}m R S \geq 2 \\
n=32\end{array}$ & $p$-value \\
\hline Age, years & $65.0 \pm 12.9$ & $64.0 \pm 12.8$ & $72.0 \pm 12.0$ & 0.001 \\
\hline Gender, male & $136(54.8)$ & $116(53.7)$ & $20(62.5)$ & 0.447 \\
\hline Body mass index, $\mathrm{kg} / \mathrm{m}^{2}$ & $24.0 \pm 3.2$ & $24.3 \pm 3.3$ & $22.4 \pm 2.7$ & 0.003 \\
\hline Initial NIHSS & $1.0 \pm 2.4$ & $0.7 \pm 1.7$ & $2.9 \pm 4.7$ & 0.015 \\
\hline Premorbid $\mathrm{mRS}$ & $0.2 \pm 0.8$ & $0.2 \pm 0.6$ & $0.6 \pm 1.2$ & 0.110 \\
\hline Discharge NIHSS & $0.1 \pm 0.6$ & $0.1 \pm 0.6$ & $0.1 \pm 0.5$ & 0.779 \\
\hline Discharge mRS & $0.2 \pm 0.6$ & $0.2 \pm 0.5$ & $0.3 \pm 0.7$ & 0.462 \\
\hline
\end{tabular}

Table 2. Comorbid determinants of good or less outcome (mRS $>1)$ of acute ischemic stroke or transient ischemic attack patients

\begin{tabular}{|c|c|c|c|c|c|c|c|c|}
\hline \multirow[b]{3}{*}{ Risk factors of CVD } & \multicolumn{4}{|c|}{ Acute ischemic stroke } & \multicolumn{4}{|c|}{ Transient ischemic attack } \\
\hline & \multicolumn{2}{|c|}{ Unadjusted OR (95\% Cl) } & \multicolumn{2}{|c|}{ Adjusted OR $(95 \% \mathrm{Cl})^{*}$} & \multicolumn{2}{|c|}{ Unadjusted OR $(95 \% \mathrm{Cl})$} & \multicolumn{2}{|c|}{ Adjusted OR $(95 \% \mathrm{Cl})^{*}$} \\
\hline & & & & & & & & \\
\hline Hypertension & $1.137(0.954-1.356)$ & 0.151 & $1.055(0.850-1.308)$ & 0.628 & $0.810(0.384-1.705)$ & 0.578 & $0.926(0.380-2.254)$ & 0.866 \\
\hline Diabetes mellitus & $1.168(0.986-1.384)$ & 0.072 & 1.256 (1.029-1.533) & 0.025 & $1.127(0.457-2.777)$ & 0.796 & $0.998(0.378-2.636)$ & 0.996 \\
\hline Dyslipidemia & $0.812(0.685-0.963)$ & 0.017 & $0.874(0.717-1.066)$ & 0.184 & $0.767(0.337-1.741)$ & 0.525 & $0.948(0.392-2.291)$ & 0.906 \\
\hline Atrial fibrillation & $1.518(1.230-1.874)$ & $<0.001$ & $1.042(0.758-1.432)$ & 0.800 & $1.014(0.283-3.627)$ & 0.983 & $0.738(0.185-2.946)$ & 0.668 \\
\hline Coronary heart disease & $1.187(0.871-1.618)$ & 0.277 & $1.124(0.783-1.613)$ & 0.527 & $0.432(0.055-3.389)$ & 0.425 & $0.414(0.048-3.584)$ & 0.423 \\
\hline Previous stroke or TIA & $1.717(1.431-2.062)$ & $<0.001$ & 1.523 (1.233-1.882) & $<0.001$ & $0.946(0.433-2.064)$ & 0.888 & $0.781(0.331-1.841)$ & 0.572 \\
\hline $\begin{array}{l}\text { Symptomatic carotid } \\
\text { artery disease }\end{array}$ & $1.276(0.635-2.563)$ & 0.494 & $1.123(0.499-2.526)$ & 0.779 & - & 1.000 & - & - \\
\hline Peripheral artery disease & $1.825(0.692-4.809)$ & 0.224 & $2.351(0.793-6.971)$ & 0.123 & - & 1.000 & - & - \\
\hline Family history of CVD & $1.274(0.477-3.405)$ & 0.629 & $1.683(0.524-5.402)$ & 0.382 & - & 1.000 & - & - \\
\hline \multicolumn{9}{|l|}{ Comorbid condition } \\
\hline Congestive heart failure & $1.275(0.589-2.762)$ & 0.538 & $0.911(0.343-2.422)$ & 0.852 & $14.33(1.261-162.92)$ & 0.032 & $9.687(0.715-131.16)$ & 0.088 \\
\hline Dementia & $4.974(2.684-9.218)$ & $<0.001$ & 3.389 (1.726-6.652) & $<0.001$ & - & - & - & - \\
\hline Chronic pulmonary disease & $1.373(0.815-2.313)$ & 0.233 & $1.171(0.648-2.115)$ & 0.600 & - & - & - & - \\
\hline Connective tissue disease & $0.705(0.236-2.110)$ & 0.532 & $0.504(0.144-1.764)$ & 0.284 & - & - & - & - \\
\hline Ulcer & $1.532(0.659-3.560)$ & 0.321 & $1.028(0.388-2.724)$ & 0.955 & - & - & - & - \\
\hline $\begin{array}{l}\text { Chronic liver disease or } \\
\text { cirrhosis }\end{array}$ & $1.560(0.644-3.779)$ & 0.324 & $1.356(0.454-4.050)$ & 0.585 & $6.935(0.423-113.74)$ & 0.175 & $4.118(0.236-71.95)$ & 0.332 \\
\hline $\begin{array}{l}\text { Moderate to severe } \\
\text { kidney disease }\end{array}$ & 2.265 (1.435-3.577) & $<0.001$ & $2.524(1.509-4.223)$ & $<0.001$ & $2.813(0.522-15.15)$ & 0.229 & $1.909(0.304-11.97)$ & 0.490 \\
\hline Lymphoma or leukemia & $0.954(0.213-4.270)$ & 0.951 & $1.026(0.187-5.643)$ & 0.976 & - & - & - & - \\
\hline Solid tumor & $2.281(1.684-3.090)$ & $<0.001$ & $1.852(1.300-2.637)$ & 0.001 & $1.242(0.262-5.881)$ & 0.784 & $1.163(0.201-6.721)$ & 0.866 \\
\hline Metastatic tumor & 8.733 (4.466-17.08) & $<0.001$ & 6.446 (3.091-13.44) & $<0.001$ & - & - & - & - \\
\hline Charlson comorbidity index & $1.345(1.265-1.430)$ & $<0.001$ & $1.294(1.206-1.388)$ & $<0.001$ & $1.005(0.729-1.387)$ & 0.975 & $0.929(0.644-1.338)$ & 0.692 \\
\hline
\end{tabular}

*Adjusted for age, gender, body mass index, ischemic stroke classification, and initial

NIHSS

\section{CONCLUSIONS}

This study demonstrated that multiple comorbidity has a significant impact on functional outcome at 3 months in patient with AIS, but not in those with TIA. This suggests that treatment of comorbid conditions is important as well as the index stroke itself. 